\title{
Effect of dormancy on the development of phloem fiber clusters
}

\author{
Kei'ichi Baba ${ }^{* *}$, Yuko Kurita ${ }^{2}$ and Tetsuro Mimura ${ }^{3}$
}

\begin{abstract}
The development of phloem fibers was investigated in poplars grown under artificially fixed conditions and under an artificially shortened annual cycle system that includes a dormancy phase. The phloem tissues in these trees formed two- or three-layer fiber clusters; however, clusters in trees that underwent periodic dormancy were larger than those in trees that did not. Individual fibers were also larger in the former than in the latter, as assessed on transverse sections. Differences were most pronounced in the radial diameter of fibers in the layer nearest the cambium. These results suggest that dormancy affects both the size of fiber clusters and the diameter of individual fibers. Furthermore, these results imply that dormancy affects the development of phloem fibers, and that the shortened annual cycle system is a useful model for investigating phloem development caused by dormancy repetition.
\end{abstract}

Keywords: Annual rhythm, Phloem fiber, Maturation, Dormancy, Poplar (Populus)

\section{Introduction}

Although fibers are well known to form in the secondary phloem, their structure varies among species. Accordingly, Esau [1] classified phloem fibers as separated single strands or as clustered parallel and tangential bands. Similarly, Furuno [2] investigated the bark in 55 different Japanese hardwoods and classified phloem fibers as diffuse, banded, and clustered. Of note, some phloem fibers, such as those from hemp, jute, flax, kenaf, ramie, and others, are commercially exploited [3]. Traditional Japanese paper is also derived from phloem fibers [4]. Nevertheless, the development of phloem fibers in living tree trunks is poorly understood.

In this study, we investigated phloem fibers in trees grown in an artificially shortened annual cycle that includes dormancy once a cycle, as well as in trees grown in conditions without dormancy, i.e., under constant conditions in a growth room. As all trees were grown for the same duration, any tissues formed are directly comparable without correction or adjustment. We have previously established a shortened annual cycle system for poplar trees using growth chambers [5]. This system

\footnotetext{
*Correspondence: kbaba@rish.kyoto-u.ac.jp

${ }^{1}$ Research Institute for Sustainable Humanosphere, Kyoto University,

Uji 611-0011, Japan

Full list of author information is available at the end of the article
}

comprises three stages and includes dormancy and dormancy breaks every 4-5 months. Growth rings in the xylem are formed when poplars are grown over multiple cycles in this system [6], but not in poplars grown without periodic dormancy.

\section{Results and discussion}

Cuttings of poplar were grown over three shortened annual cycles, with one dormancy phase per cycle. Trees were harvested at the end of the third dormancy. As a negative control, other cuttings were grown for the same duration in a fixed condition without a dormancy phase. Notably, stem diameter was comparable between the conditions with and without dormancy, while the phloem was marginally thinner in trees grown without dormancy than in trees grown in a shortened annual cycle system (Tables 1, 2, 3). Nevertheless, phloem fibers formed in 2-3 layers in all trees. Neither stem diameter nor phloem thickness was correlated with the number of fiber layers.

However, the size of fiber clusters and the distance between such clusters were quite different among trees grown with or without dormancy phases (Fig. 1). Differences were especially pronounced in layers nearest to the cambium. In a field-grown tree (Fig. 1c), phloem fibers nearest to the cambium formed tangential bands separated almost only by ray parenchyma. Fiber bundles in 
Table 1 Stem diameter and phloem thickness

\begin{tabular}{|c|c|c|c|c|c|c|}
\hline \multirow{2}{*}{$\begin{array}{l}\text { Treatment } \\
\text { Tree \#: }\end{array}$} & \multicolumn{3}{|c|}{ Without dormancy } & \multicolumn{3}{|c|}{ With dormancy } \\
\hline & $\mathrm{N}-1$ & $\mathrm{~N}-2$ & $\mathrm{~N}-3$ & $\mathrm{D}-1$ & D-2 & $D-3$ \\
\hline Stem diameter (mm) & $5.55 \pm 0.08$ & $5.95 \pm 0.20$ & $5.98 \pm 0.06$ & $6.41 \pm 0.09$ & $5.41 \pm 0.09$ & $5.45 \pm 0.06$ \\
\hline Phloem thickness (mm) & $0.60 \pm 0.05$ & $0.67 \pm 0.03$ & $0.76 \pm 0.05$ & $0.78 \pm 0.04$ & $0.78 \pm 0.06$ & $0.78 \pm 0.05$ \\
\hline Fiber layers & 2 & 2 & 3 & 3 & 2 & 3 \\
\hline
\end{tabular}

Data are mean $\pm S D$

Table 2 Comparison of stem diameter

\begin{tabular}{lll}
\hline Tree \# & Mean & Sign \\
\hline D-1 & 6.41 & a \\
N-3 & 5.98 & $\mathrm{~b}$ \\
$\mathrm{~N}-2$ & 5.95 & $\mathrm{~b}$ \\
$\mathrm{~N}-1$ & 5.55 & $\mathrm{C}$ \\
$\mathrm{D}-2$ & 5.41 & $\mathrm{c}$ \\
$\mathrm{D}-3$ & 5.45 & $\mathrm{c}$ \\
\hline
\end{tabular}

Different signs indicate significant difference. All $p<0.01$ after Bonferroni correction

Table 3 Comparison of phloem thickness

\begin{tabular}{lll}
\hline Tree \# & Mean & Sign \\
\hline D-3 & 0.78 & $\mathrm{a}$ \\
D-1 & 0.78 & $\mathrm{a}$ \\
$\mathrm{D}-2$ & 0.78 & $\mathrm{a}$ \\
$\mathrm{N}-3$ & 0.76 & $\mathrm{a}$ \\
$\mathrm{N}-2$ & 0.67 & $\mathrm{~b}$ \\
$\mathrm{~N}-1$ & 0.60 & $\mathrm{c}$ \\
\hline
\end{tabular}

Different signs indicate significant difference. All $p<0.01$, except between $\mathrm{N}-1$ and N-2 $(p<0.05)$, after Bonferroni correction

trees grown in a shortened annual cycle system (Fig. 1b) were similar in structure, but were shorter along the tangential length, and were sometimes separated by both axial and ray parenchyma. In contrast, fiber clusters in trees growing without a dormancy phase (Fig. 1a) did not form bands and were well separated by several to a dozen ray parenchyma and many axial parenchyma.

Based on cluster size and distances between clusters (Table 4), every feature, including tangential and radial sizes of fiber clusters and distance between clusters, was significantly different between trees grown with and without dormancy, especially in the first layer (Table 5). The third layer showed no significant differences in any feature. The features of the second layer were in between those of the first and third layers.

As seen in transverse micrographs of typical fiber clusters (Fig. 2), individual fibers were observed to gradually

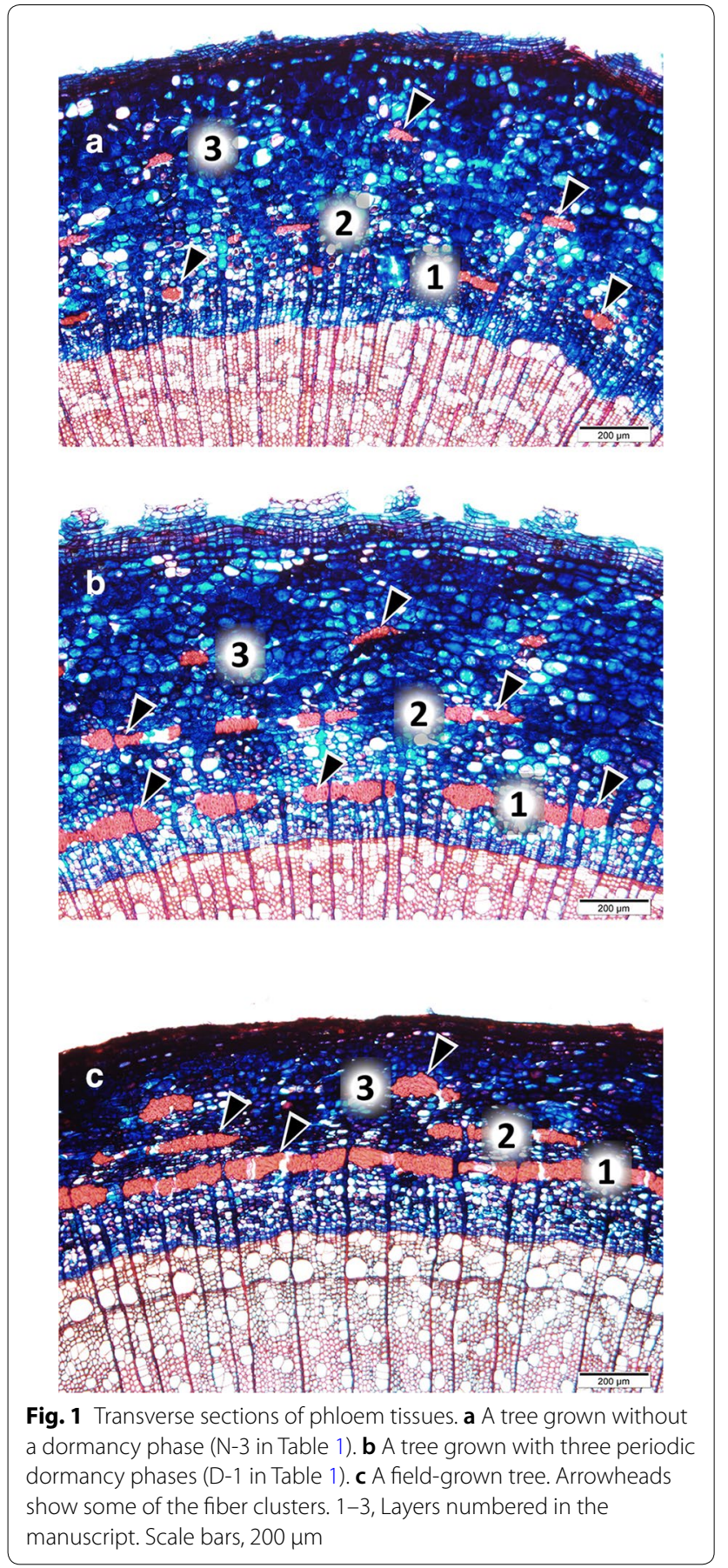


Table 4 Size of and distance between fiber clusters

\begin{tabular}{|c|c|c|c|c|c|c|}
\hline \multirow{2}{*}{$\begin{array}{l}\text { Treatment } \\
\text { Layer: }\end{array}$} & \multicolumn{3}{|c|}{ Without dormancy } & \multicolumn{3}{|c|}{ With dormancy } \\
\hline & $1 \mathrm{st}$ & 2nd & 3rd & $1 \mathrm{st}$ & 2nd & 3rd \\
\hline Tangential length ( $\mu \mathrm{m})$ & $103 \pm 58$ & $97 \pm 48$ & $96 \pm 35$ & $206 \pm 110$ & $120 \pm 62$ & $102 \pm 38$ \\
\hline Radial length ( $\mu \mathrm{m})$ & $38 \pm 9$ & $28 \pm 12$ & $28 \pm 7$ & $61 \pm 14$ & $41 \pm 9$ & $34 \pm 8$ \\
\hline Distance between clusters ( $\mu \mathrm{m})$ & $354 \pm 213$ & $455 \pm 226$ & $542 \pm 210$ & $115 \pm 64$ & $296 \pm 161$ & $509 \pm 196$ \\
\hline
\end{tabular}

Layers were numbered from cambium to epidermis. Data are mean $\pm S D$

Table 5 Comparison between trees with and without dormancy

\begin{tabular}{lll}
\hline & Layer & $\boldsymbol{p}$ value \\
\hline Tan & L1 & $0.0001^{* *}$ \\
& L2 & 0.1298 \\
Rad & L3 & 0.7092 \\
& L1 & $0.0000^{* *}$ \\
Dist & L3 & $0.0001^{* *}$ \\
& L1 & 0.0453 \\
& L2 & $0.0000^{* *}$ \\
& L3 & $0.0045^{* *}$ \\
\hline
\end{tabular}

Tan tangential length, Rad radial length, Dist distance between clusters. Values represent results of $t$ test

** $p<0.01$

increase in diameter with proximity to the cambium in all trees, also becoming less round and more radially elongated polygonal. We note that based on definitions provided by the International Association of Wood Anatomists, Populus alba L. is rounded polygonal type [7]. The diameter of individual fibers was different between trees with and without dormancy, and the radial and tangential diameters were measured (Table 6). Based on the mean values, the fibers in the third layer of trees without dormancy appeared slightly larger than those of trees with dormancy, but the difference was not statistically significant. The radial diameter in the first layer of trees without dormancy was significantly smaller than that in the other conditions (Table 7). The other diameters were not significantly different among the growing conditions. These results implied that the aspect ratio in transverse sections is much larger in trees grown with three periodic dormancy phases, especially in the first layer. Fibers produced in a field-grown tree were similar in size and structure to those in trees grown in an artificially shortened cycle system, with the aspect ratio gradually increasing with distance from the cambium. Indeed, the aspect ratio was almost the same in the second and third layers from the cambium, but smaller in the first layer.

Fiber formation in the shortened annual cycle system was observed (Fig. 3). The transverse sections were obtained from three areas of a tree grown with three periodic dormancy phases. In the top area, the tissue contained a single growth ring of wood, and the phloem fibers were formed in a single layer surrounding the whole phloem (Fig. 3a, d). The middle area contained two growth rings of wood and newly formed phloem fibers at the top-right half, inside the layer that surrounded the whole phloem (Fig. 3b, e, f). The bottom area, which consisted of three rings of wood, showed one newly formed cluster inside the two layers, both of which had already been formed in phloem surrounding the stem (Fig. 3c, g). These results indicated that the outermost layer was formed before a dormancy phase, but the inner layers were formed after at least one dormancy phase.

Collectively, the data indicated that the size and shape of individual and clustered fibers are influenced by cycles of dormancy, but not by length of culture, stem diameter, or phloem thickness. The fiber sizes in the layer nearest the cambium varied the most between trees with and without dormancy. The fibers in this layer formed from the cambium, which underwent dormancy in the shortened cycle but not in the condition without dormancy.

In a previous study, branch number increased with dormancy phases under the same shortened annual cycle system, but no branches developed on trees grown without a dormancy phase [8]. Generally, within the same species, a higher branch number is indicative of an older tree. The phenomena of branching and phloem development suggest that dormancy accelerates the maturation of trees.

\section{Conclusion}

Dormancy affects the development of individual phloem fibers and fiber clusters. However, no dormancy inhibits the development of phloem fibers and their clusters. The shortened annual cycle system is a useful model for investigating the maturation of phloem tissues.

\section{Methods \\ Plant materials}

Ramets of the same poplar (P. alba) clone were prepared as previously reported [6]. Briefly, cuttings of $3-5 \mathrm{~cm}$ length were planted in pots $(7.5 \mathrm{~cm}$ diameter, $6.5 \mathrm{~cm}$ depth) with soil and watered until rooting. After new shoots were observed at axillary buds, the cuttings were supplemented with Hyponex fertilizer (N/P/K 6:10:5, 


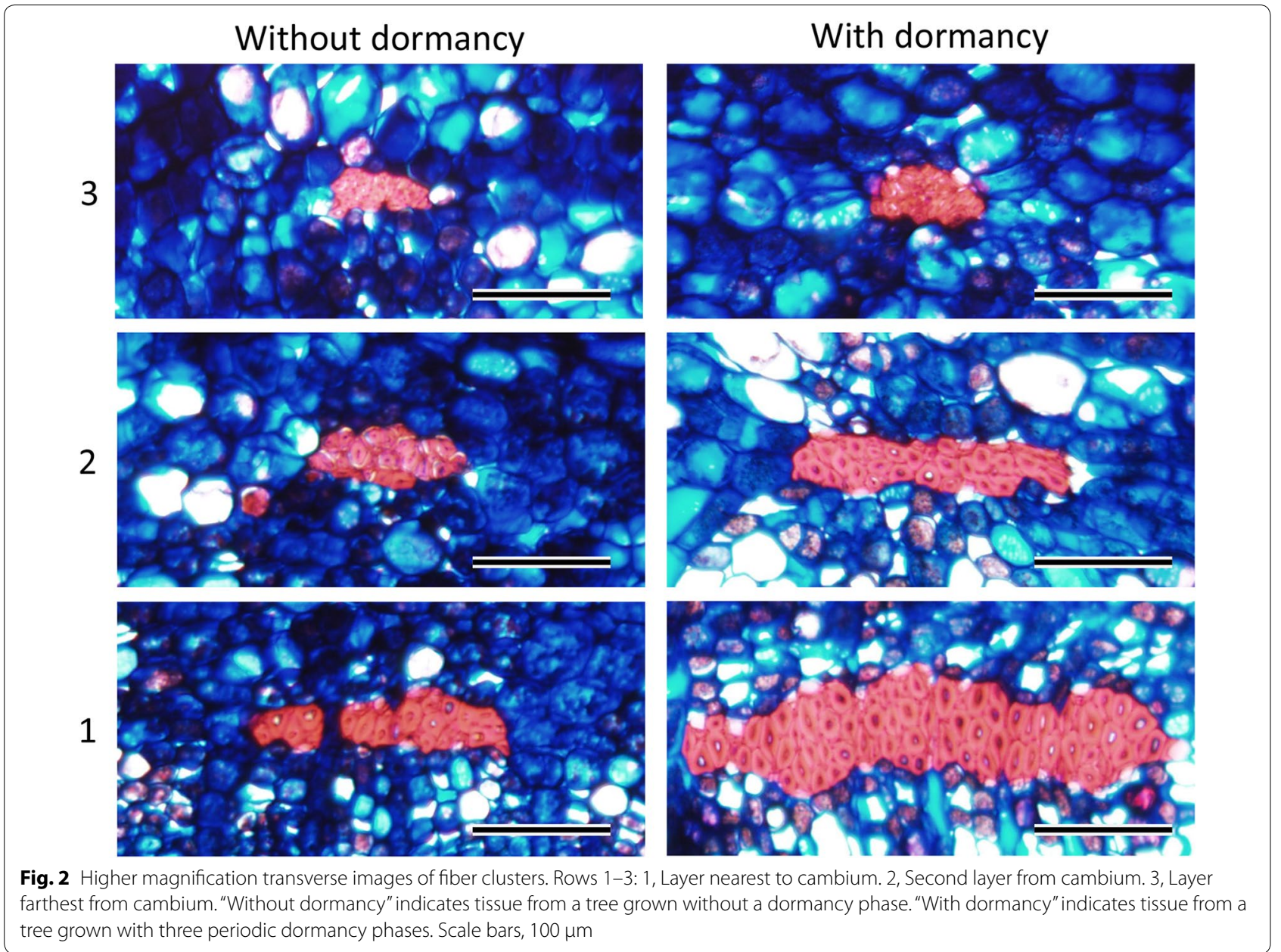

Table 6 Transverse shape of individual fibers

\begin{tabular}{|c|c|c|c|c|c|c|c|c|c|}
\hline \multirow{2}{*}{$\begin{array}{l}\text { Treatment } \\
\text { Layer: }\end{array}$} & \multicolumn{3}{|c|}{ Without dormancy } & \multicolumn{3}{|c|}{ With dormancy } & \multicolumn{3}{|l|}{ Field } \\
\hline & $1 \mathrm{st}$ & 2nd & $3 r d$ & $1 \mathrm{st}$ & 2nd & $3 r d$ & $1 \mathrm{st}$ & 2nd & $3 r d$ \\
\hline $\operatorname{Tan}(\mu \mathrm{m})$ & $14.2 \pm 2.3$ & $12.7 \pm 3.4$ & $13.2 \pm 2.6$ & $13.6 \pm 2.9$ & $14.6 \pm 2.2$ & $11.0 \pm 2.3$ & $16.3 \pm 2.5$ & $14.6 \pm 1.5$ & $13.9 \pm 2.9$ \\
\hline $\operatorname{Rad}(\mu \mathrm{m})$ & $19.3 \pm 3.3$ & $13.8 \pm 2.3$ & $12.0 \pm 2.6$ & $26.3 \pm 6.3$ & $15.4 \pm 3.1$ & $11.2 \pm 1.5$ & $27.0 \pm 5.7$ & $16.1 \pm 2.6$ & $10.4 \pm 1.9$ \\
\hline Ratio & $1.4 \pm 0.3$ & $1.2 \pm 0.5$ & $0.9 \pm 0.3$ & $2.0 \pm 0.6$ & $1.1 \pm 0.4$ & $1.1 \pm 0.3$ & $1.7 \pm 0.3$ & $1.1 \pm 0.3$ & $0.8 \pm 0.2$ \\
\hline
\end{tabular}

Layers were numbered from cambium to epidermis

Tan tangential diameter of a fiber, Rad radial diameter of a fiber, Ratio aspect ratio of the fiber section (mean \pm SD)

Table 7 Comparison of radial diameter of fibers in first layer

\begin{tabular}{ll}
\hline Comparison & $\boldsymbol{p}$ value \\
\hline W/O-W/ & $0.0065^{* *}$ \\
W/O-F & $0.0017^{* *}$ \\
W/-F & 0.8013 \\
\hline
\end{tabular}

W/ with dormancy, W/O without dormancy, F field-grown. Aspect ratio was not compared. Values represent results of $t$ test with Bonferroni correction ** $p<0.01$
HYPONeX Japan, Osaka, Japan) diluted 2000-fold. Three individual poplar trees were cultured over three artificially shortened annual cycles as described below. A 3 -year-old sucker in a field-grown tree at the Uji campus of Kyoto University was used for comparison.

\section{Growth in a shortened annual cycle system}

As previously described [5], the shortened annual cycle consisted of stage 1 (14-h light, 10 -h dark, $24-28{ }^{\circ} \mathrm{C}$ ), 

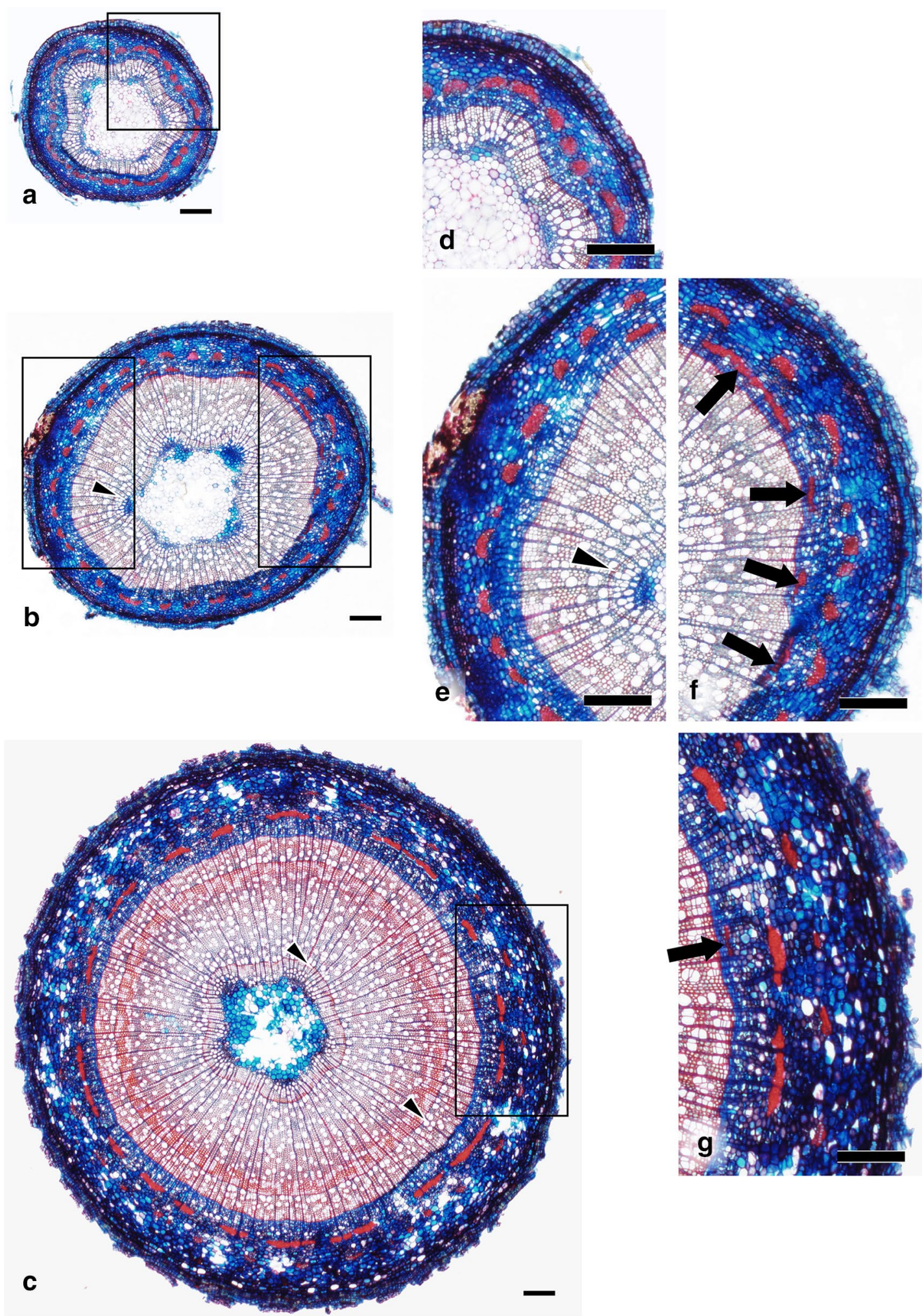

Fig. 3 Formation of fiber clusters in different growth cycles. The tissues were harvested from different parts of a tree grown with three periodic dormancy phases. a Top area with single growth ring of wood. $\mathbf{b}$ Middle area with two growth rings of wood. $\mathbf{c}$ Bottom area with three growth rings of wood. $\mathbf{d}-\mathbf{g}$ Magnified images of boxed areas in $\mathbf{a}-\mathbf{c} ; \mathbf{d}$ shows the upper-right area in $\mathbf{a}$, e shows the left area in $\mathbf{b}$, $\mathbf{f}$ shows the right area in $\mathbf{b}$, and $\mathbf{g}$ shows the right area in $\mathbf{c}$. Arrowheads show the boundaries of growth rings. Arrows show the newly formed fiber clusters. Scale bars, $200 \mu \mathrm{m}$ 
stage 2 (8-h light, 16 -h dark, $15^{\circ} \mathrm{C}$ ), and stage 3 (8-h light, 16 -h dark, $\left.5^{\circ} \mathrm{C}\right)$. Stages 1 and 2 were set at 1 month each, while the duration of stage 3 was determined based on visual assessment of leaves for, e.g., easy detachment from the stem owing to the formation of the abscission layer. Therefore, stage 3 was usually longer, lasting 2-3 months. Three cuttings prepared at the same time as test samples were grown in fixed conditions identical to stage 1.

\section{Microscopy}

Samples were excised approximately $5 \mathrm{~cm}$ from the base of a newly growing stem and fixed in 5\% formalin, 5\% acetic acid, and $40 \%$ ethanol until use. Transverse $25 \mu \mathrm{m}$ sections were prepared using a sliding microtome and stained with $1 \%$ Safranin and $1 \%$ Astra Blue. After dehydrating over an ethanol series, sections were soaked in xylene twice and mounted on glass slides with Biolite (Okenshoji, Tokyo, Japan). For observation of fiber formation, stem tissue was excised from the top, middle, and bottom part of a tree in the third dormancy phase and prepared as described above. The cycle during which the tissue formed was confirmed by counting the number of wood growth rings in the sections. These sections were examined under a BX50 light microscope (Olympus, Tokyo, Japan), and imaged with a DP73 digital camera (Olympus).

\section{Measurement and statistical analysis}

Dimensions of histological features of interest were measured in ImageJ. Each feature was measured as follows: for stem diameter and phloem thickness, three different directions of three sections; for cluster size and distance, nine areas each in three individual trees; for fiber size, ten fibers at random.

Statistical analysis was performed in Excel using $t$ tests with normal distribution. The size and distance of clusters were compared between the conditions with and without dormancy. Stem diameter, phloem thickness, and individual fiber size were subjected to multiple comparisons followed by Bonferroni's correction. Stem diameter and phloem thickness were tested among all six trees. Sizes of individual fibers were compared among three conditions of with and without dormancy and field growing.

\section{Acknowledgements}

We would like to thank Editage (http://www.editage.jp) for English language editing.

\section{Authors' contributions}

$\mathrm{KB}$ had a major role in implementing the overall work including designing the experiments, performing the required experiments, and writing the manuscript. YK contributed to designing plant cultures. TM discussed the results and contributed to the conclusions. All authors read and approved the final manuscript.

\section{Funding}

This work was supported by JSPS KAKENHI Grant Number JP18K05761 and the Research Institute for Sustainable Humanosphere, Kyoto University (Mission 1).

\section{Availability of data and materials}

The datasets used and/or analyzed during the current study are available from the corresponding author on reasonable request.

\section{Competing interests}

The authors declare that they have no competing interests.

\section{Author details}

${ }^{1}$ Research Institute for Sustainable Humanosphere, Kyoto University, Uji 611-0011, Japan. ${ }^{2}$ Faculty of Agriculture, Ryukoku University, Otsu 520-2194, Japan. ${ }^{3}$ Graduate School of Science, Kobe University, Kobe 657-8501, Japan.

Received: 3 March 2019 Accepted: 31 July 2019

Published online: 09 August 2019

\section{References}

1. Esau K (1977) Phloem. Anatomy of seed plants, 2nd edn. Wiley, New York, pp 157-181

2. Furuno $T$ (1990) Bark structure of deciduous broad-leaved trees grown in the San'in region, Japan. IAWA Bull 11(3):239-254

3. Papadopouloua E, Bikiarisb D, Chrysafisc K, Wladyka-Przybylakd M, Wesolekd D, Mankowskid J, Kolodziejd J, Baranieckid P, Bujnowiczd K, Gronberg V (2015) Value-added industrial products from bast fiber crops. Ind Crops Prod 68:116-125

4. Masuda K (1985) Japanese paper and Hyogu. Paper Conserv 9(1):32-41

5. Kurita Y, Baba K, Ohnishi M, Anegawa A, Shichijo C, Kosuge K, Fukaki H, Mimura T (2014) Establishment of a shortened annual cycle system; a tool for the analysis of annual re-translocation of phosphorus in the deciduous woody plant (Populus alba L.). J Plant Res 127(4):545-551

6. Baba K, Kurita Y, Mimura T (2018) Wood structure of Populus alba formed in a shortened annual cycle system. J Wood Sci 64(1):1-5

7. Angyalossy V, Pace MR, Evert RF, Marcati CR, Oskolski AA, Terrazas T, Kotina E, Lens F, Mazzoni-Viveiros SC, Angeles G, Machado SR, Crivellaro A, Rao KS, Junikka L, Nikolaeva N, Baas P (2016) IAWA list of microscopic bark features. IAWA J 37(4):517-615

8. Baba K, Kurita Y, Mimura T (2017) Architectural morphogenesis of poplar grown in a shortened annual cycle system. Sustain Humanosphere 13:1-4

\section{Publisher's Note}

Springer Nature remains neutral with regard to jurisdictional claims in published maps and institutional affiliations. 